\title{
A comparative study of luteal estradiol pre-treatment in GnRH antagonist protocols and in micro dose flare protocols for poor-responding patients
}

\author{
Robab Davar - Mozhgan Rahsepar • \\ Elham Rahmani
}

Received: 20 April 2012/Accepted: 9 August 2012/Published online: 26 August 2012

(C) Springer-Verlag 2012

\begin{abstract}
Purpose This study aims to verify if luteal estradiol pretreatment improves IVF/ICSI outcomes in a GnRH antagonist protocol as compared with a micro dose GnRH agonist protocol in poor-responding patients.

Methods A total of 116 IVF/ICSI cycles were included in this prospective randomized single blind clinical trial. The selected women were randomly assigned to receive an estradiol pre-treatment in a GnRH antagonist protocol (daily oral Estradiol Valerate $4 \mathrm{mg}$ preceding the IVF cycle from the 21st day until the first day of the next cycle) or in oral contraceptive pill micro dose GnRH agonist protocol. Results The patients in the luteal estradiol protocol required more days of stimulation $(10.9 \pm 1.6$ vs. $10.2 \pm$ $1.8)$ and a greater gonadotropin requirement $(3,247.8 \pm$ 634.6 vs. $2,994.8 \pm 611 \mathrm{IU})$, yet similar numbers of oocytes were retrieved and fertilized. There was no significant difference between the two groups in terms of the implantation rates $(9.8$ vs. $7.9 \%)$ and the clinical pregnancy rates per transfer (16.3 vs. $15.6 \%$ ).

Conclusion This study demonstrates that the use of estradiol during a preceding luteal phase in a GnRH antagonist protocol can provide similar IVF outcomes when compared to a micro dose GnRH agonist protocol.
\end{abstract}

R. Davar · M. Rahsepar · E. Rahmani ( $₫)$

Yazd Research and Clinical Center for Infertility,

Shahid Sadoughi University of Medical Sciences,

Bouali Street, Safaieh, 8916877391 Yazd, Iran

e-mail: rahmani@bpums.ac.ir

E. Rahmani

Obstetrics and Gynecology Ward, Bushehr University

of Medical Sciences, Bushehr, Iran
Keywords Poor responders - IVF outcome . Luteal phase $\cdot$ Estradiol $\cdot$ Micro dose protocol . GnRH antagonist

\section{Introduction}

Some women undergoing infertility treatments respond poorly to the usual gonadotropin stimulation protocol [1]. In spite of significant advances in assisted reproductive techniques (ART), the management of poor-responding patients is still intricate [2]. A poor ovarian response to controlled ovarian hyper stimulation $(\mathrm{COH})$ occurs in 9-24\% of women undergoing IVF [1, 3, 4]. Although there is lack of uniform definitions, poor response to controlled ovarian hyper stimulation can be defined as insufficient ovarian response in terms of low number of follicles developed, low serum E2 levels, and low number of oocytes retrieved in spite of adequate ovarian stimulation $[1,4]$. Poor-response patients, following a standard ovarian stimulation protocol, have lower pregnancy rates compared with normal responders [1].

So far, several strategies have been recommended for poor responders. The most frequently used ovarian stimulation protocols are beginning of gonadotropin and a $\mathrm{GnRH}$ agonist (GnRH-a) together in the follicular phase (the socalled micro dose flare protocol) and the GnRH antagonist regimens [3]. A micro dose protocol has the benefit of the initial rise in endogenous gonadotropin and maintains effectiveness in premature luteinizing hormone (LH) surge prevention. Pretreatment with oral contraceptives prevents corpus luteum formation and the following stimulation from exogenous GnRH-a [5].

GnRH antagonists are interesting because their beginning occurs after the start of gonadotropin stimulation, thus 
minimizing their impact on early follicular recruitment. [6]. An approach for treating poor responders is to give E2 in the luteal phase before IVF hyper stimulation [7-9].

Endogenous FSH in the previous luteal phase may selectively stimulate larger follicles and consequently lead to a size inconsistency in the developing follicles. This size inconsistency may cause fewer follicles to be responsive to gonadotropin stimulation [10]. Fanchin et al. [8] found that luteal phase E2 resulted in a greater number of follicles $\geq 16 \mathrm{~mm}$, further mature oocytes, and more accessible embryos when compared with a control group.

Thus, the purpose of the present study is to compare the results gained from the stimulation of patients who had anticipated poor ovarian responses and got a luteal phase estradiol pre-treatment in a GnRH antagonist protocol with the results gained from the stimulation of those who underwent in a micro dose agonist flare protocol.

\section{Materials and methods}

\section{Study design}

This prospective randomized single blind clinical trial included 116 women with poor ovarian responses, who underwent $\mathrm{COH}$ for IVF in Yazd Research and Clinical Center for Infertility affiliated to Shahid Sadoughi University of Medical Sciences from March 2011 to March 2012. The study was approved by the ethics committee of Yazd Research and Clinical Center for Infertility. Randomized assignment of the two treatments was performed by a computer-based program. Before starting the study, informed consent was obtained from each couples. The inclusion criteria were a history of poor response in a prior cycle ( $\leq 3$ oocytes retrieved, poor-quality oocytes, or cycle cancelation due to inadequate ovarian response), or women anticipated to be a poor responder based on initial testing (third-day FSH level of $10 \mathrm{mIU} / \mathrm{mL}$, or a basal antral follicle count $<5$ ). The exclusion criteria were stage III-IV endometriosis, autoimmune or chromosomal disorders, endocrine or metabolic diseases, or existence of only one ovary. Patients exhibiting a day 3 serum FSH level greater than $15 \mathrm{mIU} / \mathrm{mL}$ were excluded. Sever male factor (patients with azoospermia and normal morphology of sperm $<4 \%$ ) and patients with hydrosalpinx also were excluded from the study.

\section{Treatment protocols}

Fifty eight randomly selected women underwent IVF using the E2/antagonist protocol (E2/ANT group). In this group, estradiol (Aburaihan Pharmaceutical Co., Tehran, Iran) $2 \mathrm{mg}$ was started orally twice a day on the 21 st luteal day and continued until menstruation. Once menses began, estradiol was discontinued and gonadotropin stimulation was started on the 2nd day of the menstrual cycle. Gonal-F (Gonal-F, Serono, Italy) at 225-300 IU/day was initiated from the second day of menses and was adjusted according to serum E2 concentrations and the ovarian response as noted by ultrasound. When the leading follicle reached $14 \mathrm{~mm}$ in diameter, Cetrorelix (Merck-Serono Germany) $0.25 \mathrm{mg} \mathrm{SC}$ was added and continued every day until and including the day of hCG administration. Another group consisted of 58 women who underwent ovarian stimulation for IVF using micro dose agonist protocol (micro dose group). In these patients, low-dose OCP (30 mcg Ethinyl Estradiol and $0.3 \mathrm{mg}$ Norgestrel, Aburaihan Pharmaceutical Co., Tehran, Iran) was started on the 2nd day of the previous cycle for 21 days. On the second day of menstruation, Suprefact (Buserelin acetate, Aventis Pharma Deutschland, Germany) $50 \mu \mathrm{g}$ SC was started and continued twice a day until the day of hCG administration. After 2 days (on the fourth day of menstruation), Gonal-F was started at 225-300 IU/day. In these patients, like in the other group, the dose of Gonal-F was adjusted according to serum E2 concentrations and ovarian responses as noted by ultrasound.

\section{IVF procedure}

In both groups, 10,000 IU of hCG (pregnyl, Daropakhsh, Iran) was administered IM when at least two follicles reached $\geq 18 \mathrm{~mm}$ in diameter. The follicles were followed $36 \mathrm{~h}$ later by ultrasound-guided transvaginal oocyte retrieval. Follicles $\geq 14 \mathrm{~mm}$ aspirated and the physicians performing the follicular aspiration blinded to the stimulation protocol. The IVF and intracytoplasmic sperm injection (ICSI) procedures were performed, and the embryos were transferred on the third day after retrieval with a Labotect catheter (Labotect, Gotting, Germany). A good-quality embryo was defined as seven or more blastomeres on day 3 , equally sized blastomeres, and $<20 \%$ fragmentation and poor-quality embryos consist of all the rest. The number of transferred embryos depended on the embryo quality and the patient's age. All the patients received $100 \mathrm{mg}$ of progesterone (Aburaihan Pharmaceutical Co., Tehran, Iran) IM per day for luteal support, which was initiated on the day of oocyte retrieval. Serum B-hCG was checked 14 days after the embryo transfer. If the patient was pregnant, then progesterone was continued until the 10th week of pregnancy. Chemical pregnancy was defined as serum B-hCG $>50 \mathrm{IU} / \mathrm{L}$ after 14 days from embryo transfer. Clinical pregnancy was defined as the presence of a gestational sac with heart beat identified by ultrasound 5 weeks after the embryo transfer. The implantation rate was defined as the ratio of gestational 
sacs to the number of embryos transferred and Clinical abortion rate was determined as clinically recognized pregnancy losses before 20 weeks of gestation. Criteria for cycle cancelation due to poor ovarian response included the presence of fewer than two growing follicles on ultrasound' with an E2 level $<200 \mathrm{pg} / \mathrm{ml}$ on day 7 of stimulation.

Outcome measures and statistical analysis

The primary outcome measures included the clinical pregnancy rates and the number of oocytes retrieved. The secondary outcome measures included the cycle length, the total dose of gonadotropin, and the implantation rate. The SPSS 19 package program was used to perform all the statistical analyses. $\chi^{2}$-test, Fisher's exact test, MannWhitney test, and Independent sample $t$ test were used when appropriate. $P<0.05$ was considered statistically significant. The data are presented as the mean \pm standard deviation unless otherwise indicated.

\section{Results}

116 patients were enrolled in this study, and none of them was lost to follow up; therefore, final analysis was done on 58 patients in each group. Of the 116 poor-responding patients included in this study, 58 were treated with a micro dose protocol (MD), and 58 received estradiol pre-treatment with an antagonist protocol (E2/ANT). 12 women in E2/ANT group did not transfer embryo: three patients because of poor ovarian response, five patients because of failed oocyte retrieval, and four because of failed fertilization. In MD group, 15 women did not transfer embryo: two patients because of poor ovarian response,six patients

Table 1 Patients characteristics

\begin{tabular}{llll}
\hline Characteristics & $\begin{array}{l}\text { E2/ANT } \\
n=58\end{array}$ & $\begin{array}{l}\text { MD } \\
n=58\end{array}$ & $P$ value \\
\hline Age (years) & $32.9 \pm 6.3$ & $33.3 \pm 5.5$ & 0.72 \\
Basal FSH (IU/L) & $7.4 \pm 2.0$ & $7.8 \pm 1.8$ & 0.22 \\
Duration of infertility (years) & $7.8 \pm 5.7$ & $8.2 \pm 5.1$ & 0.43 \\
No. of prior attempted cycles & $1.5 \pm 0.6$ & $1.4 \pm 0.4$ & 0.67 \\
Etiology of infertility no (\%) & & & 0.84 \\
Male factor & $31(53.4)$ & $29(50)$ & \\
Tubal factor & $6(10.4)$ & $8(13.8)$ & \\
Unexplained & $16(27.6)$ & $14(24.1)$ & \\
Mild endometriosis & $5(8.6)$ & $7(12.1)$ & \\
\hline
\end{tabular}

Student $t$ test, Mann-Whitney test and Chi-square test. PV $<0.05$ was significant

ANT gonadotropin-releasing hormone antagonist, E2 estradiol, $M D$ micro dose because of failed oocyte retrieval, and seven because of failed fertilization. However, they were included in the final analysis. Table 1 presents the patients' characteristics in the two groups. The demographic parameters were similar in both groups, including age, basal FSH level, the number of previous cycles, etiology of infertility, and infertility duration. Table 2 compares the cycle characteristics in the two groups. The patients undergoing stimulation with E2/ANT had a significantly greater requirement for gonadotropin $(3,247.8 \pm 634.6$ vs. $2,994.8 \pm 611 \mathrm{IU}$; $P=0.03)$. As compared with MD group, they achieved a similar number of oocytes retrieved and the number of mature oocytes as well. The endometrial thickness and the estradiol concentration were similar between the two groups on the day of hCG. However, the days of stimulation in E2/ANT group were $10.9 \pm 1.6$ vs. $10.2 \pm$ 1.8 days in MD group $(P=0.04)$. The embryo data and the clinical outcome of the study groups are compared in Table 3. The number of embryos obtained and transferred and the number of good-quality embryos were not different in the two groups. In addition, no significant differences were noted in the implantation rate $(9.8$ vs. $7.9 \%$; $P=0.36)$, clinical pregnancy rate per cycle (13.8 vs. $12.1 \% ; P=0.78)$, and clinical pregnancy rate per transfer (16.3 vs. $15.6 \% ; P=0.91$ ). Also there was no difference between the two groups with regard to fertilization rate (59.7 vs. $49.6 \% ; P=0.13$ ) and clinical abortion rate $(12.5$ vs. $14.3 \% ; P=0.91)$. The percentage of conventional IVF and ICSI was similar in two groups. The total cancelation rate was not significantly different between groups. (20.7 vs. $25.8 \% ; P=0.66$ ).

Table 2 Cycle characteristics of the study groups

\begin{tabular}{lrrr}
\hline Characteristics & \multicolumn{1}{l}{$\begin{array}{l}\text { E2/ANT } \\
n=58\end{array}$} & \multicolumn{1}{l}{$\begin{array}{l}\text { MD } \\
n=58\end{array}$} & \\
\hline $\begin{array}{l}\text { No. of follicles } \\
\quad \text { 16 mm }\end{array}$ & $6.1 \pm 2.0$ & $5.7 \pm 2.5$ & 0.31 \\
$\begin{array}{l}\text { No. of oocytes } \\
\text { retrieved }\end{array}$ & $4.2 \pm 3.0$ & $3.9 \pm 2.9$ & 0.66 \\
$\begin{array}{l}\text { No. of mature } \\
\text { oocytes }\end{array}$ & $3.5 \pm 2.2$ & $3.4 \pm 2.4$ & 0.88 \\
$\begin{array}{l}\text { Days of stimulation } \\
\text { Endometrial } \\
\text { thickness on day of } \\
\text { hCG (mm) }\end{array}$ & $10.9 \pm 1.6$ & $10.2 \pm 1.8$ & 0.04 \\
$\begin{array}{l}\text { Peak E2 on day of } \\
\text { hCG (pg/ml) }\end{array}$ & $958.1 \pm 538.7$ & $943.1 \pm 563.4$ & 0.53 \\
$\begin{array}{l}\text { Total dose of } \\
\text { gonadotropin (IU) }\end{array}$ & $3,247.8 \pm 634.6$ & $2,994.8 \pm 611$ & 0.03 \\
\hline
\end{tabular}

$A N T$ gonadotropin-releasing hormone antagonist, E2 estradiol, $M D$ micro dose. Mann-Whitney test and student $t$ test. PV $<0.05$ was significant 
Table 3 Embryo data and clinical outcome of the study group

\begin{tabular}{|c|c|c|c|}
\hline Characteristics & $\begin{array}{l}\text { E2/ANT } \\
n=58\end{array}$ & $\begin{array}{l}\mathrm{MD} \\
n=58\end{array}$ & $P$ value \\
\hline No. of embryos obtained & $2.2 \pm 1.8$ & $2.0 \pm 1.9$ & 0.23 \\
\hline No. of embryos transferred & $1.6 \pm 1.0$ & $1.4 \pm 1.2$ & 0.26 \\
\hline $\begin{array}{l}\text { Transfers with good quality } \\
\text { embryos }\end{array}$ & $53.4 \%$ & $47.4 \%$ & 0.36 \\
\hline Fertilization rate & $59.7 \%$ & $49.6 \%$ & 0.13 \\
\hline Fertilization procedures $(\%)$ & & & 0.30 \\
\hline IVF & $34.5 \%$ & $24.1 \%$ & \\
\hline ICSI & $65.5 \%$ & $75.9 \%$ & \\
\hline Total cancelation rate $n(\%)$ & & & 0.66 \\
\hline Poor ovarian response & $3(5.2 \%)$ & $2(3.4 \%)$ & \\
\hline Failed oocyte retrieval & $5(8.6 \%)$ & 6 & \\
\hline Failed fertilization & $4(6.9 \%)$ & ${ }^{7}(12.1 \%)$ & \\
\hline Implantation rate & $9.8 \%$ & $7.9 \%$ & 0.36 \\
\hline Clinical pregnancy rate/cycle & $13.8 \%$ & $12.1 \%$ & 0.78 \\
\hline Clinical pregnancy rate/transfer & $16.3 \%$ & $15.6 \%$ & 0.91 \\
\hline Clinical abortion rate & $12.5 \%$ & $14.3 \%$ & 0.91 \\
\hline
\end{tabular}

ANT gonadotropin-releasing hormone antagonist, E2 estradiol, $M D$ micro dose. Mann-Whitney test and Chi-square test. PV $<0.05$ was significant

\section{Discussion}

The definition of poor-responding patients is controversial in the literature [11]. The best stimulation protocol for poor responders should have an appropriate rate of cycle cancelation, provide the maximum number of mature oocytes, and have maximal pregnancy and delivery rates [3].

Quite recently, a better understanding of follicular development has resulted in the improvement of strategies for ovarian stimulation in poor-responding patients. It has been found that during the luteal-follicular transition, FSH protects early antral follicles from atresia and causes their growth [12]. Larger follicles are more sensitive to FSH and, therefore, begin to respond and develop during the late luteal phase [7, 9, 13, 14]. Thus, asynchronous follicular growth during controlled ovarian hyper stimulation may be an outcome of the size heterogeneities of early antral follicles during the early follicular phase [7].

Thus, this study investigated the effect of exogenously administered estradiol in the preceding luteal phase in a GnRH antagonist protocol on IVF parameters and compared to a micro dose GnRH agonist protocol in poorresponding patients. The results of this study showed no significant differences between the two groups in the mean number of oocytes, mature oocytes, fertilization, implantation, and clinical pregnancy rates. Compared with the micro dose group, the patients who were receiving luteal phase estradiol experienced an increase in the total dose of gonadotropin used as well as the days of stimulation. A probable explanation for the increased use of gonadotropin is that the inhibitory effect of estradiol on FSH in the luteal phase resulted in slower growth of the follicles once stimulation started.

This study's findings are consistent with the published literature in many regards. In a retrospective paired study by Frattarelli et al., the total dose of gonadotropin used would be increased in the luteal estradiol group. However, according to their study, giving estradiol in the luteal phase before gonadotropin stimulation seems to increase the number and the quality of embryos achieved in poor responders [15].

Weitzman et al. reported that the use of E2 patch and a $\mathrm{GnRH}$ antagonist during a former luteal phase in patients with a history of poor response could offer similar IVF outcomes when compared with the micro dose GnRH agonist protocol. They found no differences in oocyte outcome, cancelation rates, or pregnancy rates between the groups. Their findings supported the use of both protocols as viable options for poor responders [11].

Dragisic et al. used a luteal E2 patch combined with a GnRH antagonist in the luteal phase before starting gonadotropin stimulation in a GnRH antagonist protocol. They showed better outcomes, as compared to outcomes in previous cycles, for patients undergoing stimulation with the luteal E2 patch/GnRH antagonist protocol [16]. The results of their study are consistent with those of Frattarelli et al. with regard to an improved number of oocytes retrieved. However, Frattarelli et al. did not find an increase in fertilization rate. The medication type and route of administration vary among studies. Frattarelli et al. used oral estradiol, as Dragisic et al. used an estradiol patch for administration medication.

Shastri et al. compared the in vitro fertilization (IVF) results for young poor responders treated in a luteal estradiol/gonadotropin-releasing hormone antagonist protocol with the results of a micro dose protocol. The patients in the E2/antagonist group had a greater gonadotropin necessity and a lower E2 level. However, the number of oocytes retrieved and fertilized was similar in the two groups. The E2/antagonist group showed to be tending toward improved implantation rates and ongoing pregnancy rates per started cycle [17].

In a retrospective cohort study, Elassar et al. [18] showed that the coordination of follicular growth in the luteal phase is no better by using E2 and a GnRH antagonist than E2 alone in poor responders.

Based on Hill et al.'s study, giving E2 in the luteal phase before gonadotropin stimulation is associated with an increase in the dose of gonadotropin required, the peak E2 levels, and the percentage of embryos reaching the $\geq 7$-cell 
stage in patients who are poor responders during IVF. Luteal E2 was associated with improved embryo development and a trend toward improved delivery rate. Consistent with other studies, the luteal E2 protocol required a higher dose of gonadotropin with longer stimulation [10].

Ye et al. randomly assigned normal responders to either standard long GnRH agonist protocol or luteal E2 before $\mathrm{GnRH}$ antagonist cycles. They reported that the luteal E2 pre-treatment before GnRH antagonist protocol significantly increases serum LH level and incidence rate of premature LH. However, compared with long GnRH agonist protocol, no significant effect is observed on implantation, clinical pregnancy, live birth, and early pregnancy loss rates [19].

According to the results of this study, neither of the two treatment regimens had major priority over the other. In conclusion, this study demonstrates that the use of estradiol during the preceding luteal phase in a GnRH antagonist protocol can bring about similar IVF outcomes when compared with a micro dose GnRH agonist protocol.

Acknowledgments The authors are grateful to the nursing and embryology staff of the Yazd Research and Clinical Center for Infertility, Shahid Sadoughi University of Medical Sciences.

Conflict of interest None.

\section{References}

1. Tarlatzis B, Zepiridis L, Grimbizis G, Bontis J (2003) Clinical management of low ovarian response to stimulation for IVF: a systematic review. Hum Reprod Update 9(1):61. doi:10.1093/ humupd/dmg007

2. Kahraman K, Berker B, Atabekoglu CS, Sonmezer M, Cetinkaya E, Aytac R, Satiroglu H (2009) Microdose gonadotropin-releasing hormone agonist flare-up protocol versus multiple dose gonadotropin-releasing hormone antagonist protocol in poor responders undergoing intracytoplasmic sperm injectionembryo transfer cycle. Fertil Steril 91(6):2437-2444. doi: 10.1016/j.fertnstert.2008.03.057

3. Demirol A, Gurgan T (2009) Comparison of microdose flare-up and antagonist multiple-dose protocols for poor-responder patients: a randomized study. Fertil Steril 92(2):481-485. doi: 10.1016/j.fertnstert.2008.07.006

4. Surrey ES, Schoolcraft WB (2000) Evaluating strategies for improving ovarian response of the poor responder undergoing assisted reproductive techniques. Fertil Steril 73(4):667-676. doi: 10.1016/S0015-0282(99)00630-5

5. Schoolcraft W, Schlenker T, Gee M, Stevens J, Wagley L (1997) Improved controlled ovarian hyperstimulation in poor responder in vitro fertilization patients with a microdose follicle-stimulating hormone flare, growth hormone protocol. Fertil Steril 67(1): 93-97. doi:10.1016/S0015-0282(97)81862-6

6. Mahutte NG, Arici A (2007) Role of gonadotropin-releasing hormone antagonists in poor responders. Fertil Steril 87(2):241-249. doi:10.1016/j.fertnstert.2006.07.1457

7. Fanchin R, Cunha-Filho S, Schonäuer LM, Kadoch IJ, CohenBacri P, Frydman R (2003) Coordination of early antral follicles by luteal estradiol administration provides a basis for alternative controlled ovarian hyperstimulation regimens. Fertil Steril 79(2):316-321. doi:10.1016/S0015-0282(02)04574-0

8. Fanchin R, Lozano DHM, Schonauer LM, Cunha-Filho JS, Frydman R (2005) Hormonal manipulations in the luteal phase to coordinate subsequent antral follicle growth during ovarian stimulation. Reprod Biomed Online 10(6):721-728. doi:10.1016/ S1472-6483(10)61115-7

9. Fanchin R, Salomon L, Castelo-Branco A, Olivennes F, Frydman N, Frydman R (2003) Luteal estradiol pretreatment coordinates follicular growth during controlled ovarian hyperstimulation with GnRH antagonists. Hum Reprod 18(12):2698. doi:10.1093/ humrep/deg516

10. Hill MJ, McWilliams GDE, Miller KA, Scott RT, Frattarelli JL (2009) A luteal estradiol protocol for anticipated poor-responder patients may improve delivery rates. Fertil Steril 91(3):739-743. doi:10.1016/j.fertnstert.2007.12.073

11. Weitzman VN, Engmann L, DiLuigi A, Maier D, Nulsen J, Benadiva C (2009) Comparison of luteal estradiol patch and gonadotropin-releasing hormone antagonist suppression protocol before gonadotropin stimulation versus microdose gonadotropinreleasing hormone agonist protocol for patients with a history of poor in vitro fertilization outcomes. Fertil Steril 92(1):226-230. doi:10.1016/j.fertnstert.2008.04.024

12. Chun SY, Eisenhauer K, Minami S, Billig H, Perlas E, Hsueh A (1996) Hormonal regulation of apoptosis in early antral follicles: follicle-stimulating hormone as a major survival factor. Endocrinology 137(4):1447-1456. doi:10.1210/en.137.4.1447

13. Fanchin R, Castelo Branco A, Kadoch IJ, Hosny G, Bagirova M, Frydman R (2004) Premenstrual administration of gonadotropinreleasing hormone antagonist coordinates early antral follicle sizes and sets up the basis for an innovative concept of controlled ovarian hyperstimulation. Fertil Steril 81(6):1554-1559. doi: 10.1016/j.fertnstert.2003.11.037

14. Hohmann FP, Macklon NS, Fauser BCJM (2003) A randomized comparison of two ovarian stimulation protocols with gonadotropin-releasing hormone $(\mathrm{GnRH})$ antagonist cotreatment for in vitro fertilization commencing recombinant follicle-stimulating hormone on cycle day 2 or 5 with the standard long GnRH agonist protocol. J Clin Endocrinol Metab 88(1):166. doi: 10.1210/jc.2002-020788

15. Frattarelli JL, Hill MJ, McWilliams GDE, Miller KA, Bergh PA, Scott RT Jr (2008) A luteal estradiol protocol for expected poorresponders improves embryo number and quality. Fertil Steril 89(5):1118-1122. doi:10.1016/j.fertnstert.2007.05.025

16. Dragisic KG, Davis OK, Fasouliotis SJ, Rosenwaks Z (2005) Use of a luteal estradiol patch and a gonadotropin-releasing hormone antagonist suppression protocol before gonadotropin stimulation for in vitro fertilization in poor responders. Fertil Steril 84(4):1023-1026. doi:10.1016/j.fertnstert.2005.04.031

17. Shastri SM, Barbieri E, Kligman I, Schoyer KD, Davis OK, Rosenwaks Z (2011) Stimulation of the young poor responder: comparison of the luteal estradiol/gonadotropin-releasing hormone antagonist priming protocol versus oral contraceptive microdose leuprolide. Fertil Steril 95(2):592-595. doi:10.1016/ j.fertnstert.2010.10.003

18. Elassar A, Mann JS, Engmann L, Nulsen J, Benadiva C (2011) Luteal phase estradiol versus luteal phase estradiol and antagonist protocol for controlled ovarian stimulation before in vitro fertilization in poor responders. Fertil Steril 95(1):324-326. doi: 10.1016/j.fertnstert.2010.07.1058

19. Ye H, Huang G, Zeng P, Pei L (2009) IVF/ICSI outcomes between cycles with luteal estradiol (E 2) pre-treatment before $\mathrm{GnRH}$ antagonist protocol and standard long GnRH agonist protocol: a prospective and randomized study. J Assist Reprod Genet 26(2):105-111. doi:10.1007/s10815-009-9300-3 